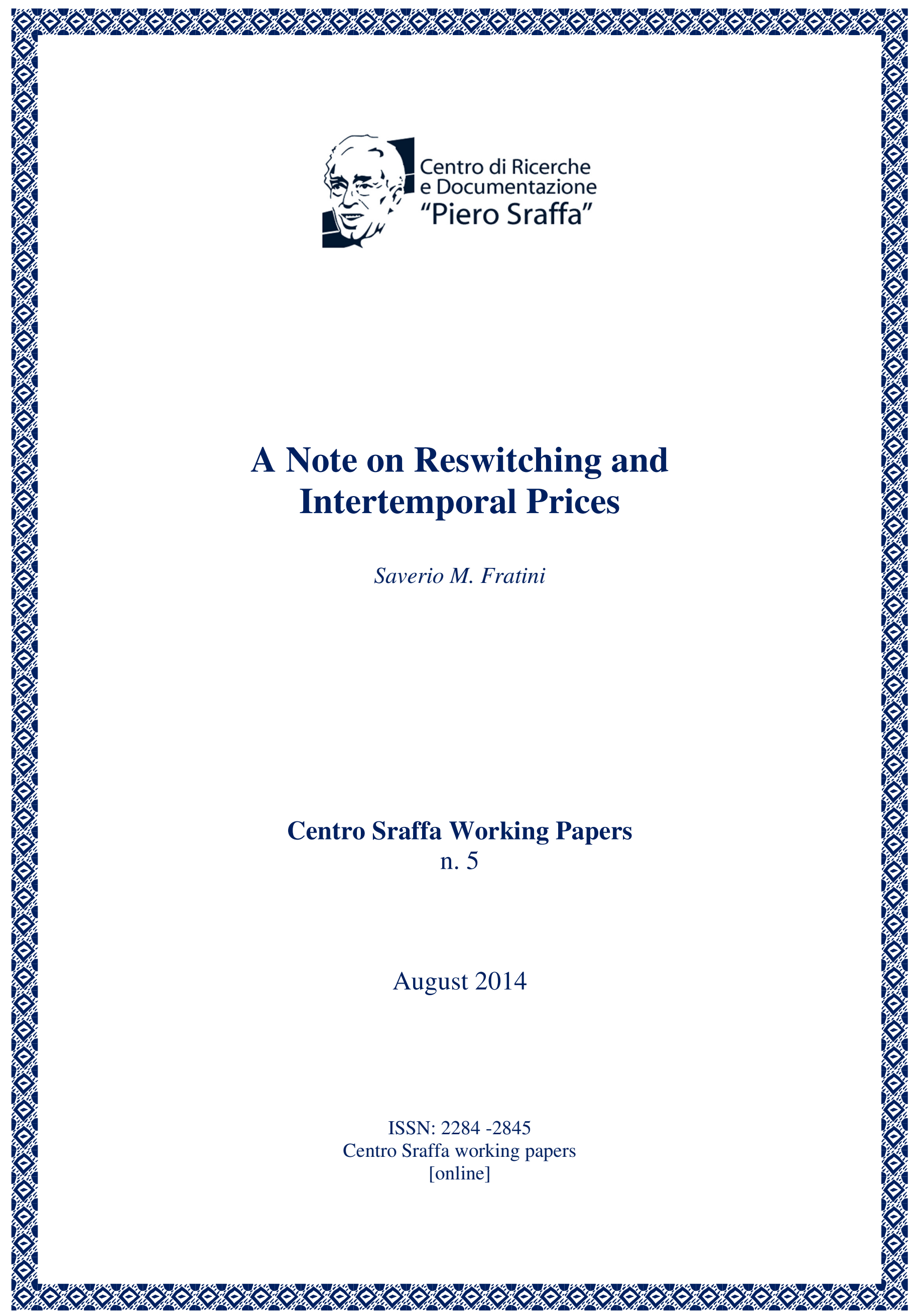




\title{
A Note on Reswitching and Intertemporal Prices
}

\author{
Saverio M. Fratini \\ Università degli Studi Roma Tre
}

\begin{abstract}
Bliss (1975) claims that reswitching is nothing but an 'optical illusion' due to the exclusion of non-stationary price sequences from the analysis. The present paper is aimed at developing this point. The standard case for choice of techniques and reswitching is reformulated in terms of Arrow-Debreu intertemporal prices and the conditions making these prices stationary are highlighted separately. It is then shown that the analysis of the choice of techniques in terms of 'switch points' requires the stationary conditions.
\end{abstract}

JEL Codes: B51, D21, D46

Keywords: reswitching, stationary prices, intertemporal prices, convex sets

\section{Introduction}

Bliss showed in his seminal work Capital Theory and the Distribution of Income (1975) that the same consumption-and-accumulation path can be optimal for more than one intertemporal price system (cf. Bliss 1975, pp. 235-44). In this connection, he also claimed that reswitching - the possibility of the same production technique being in use for interest rates lower than $\hat{r}$ and higher than $\breve{r}$ but not for $r \in(\widehat{r}, \breve{r})$ - arises when attention is confined to 'constant-rate-of- 
interest price systems' and disappears when price sequences not of this type are admitted. As he wrote,

if a sequence of activities is an equilibrium for two price sequences, then it is an equilibrium for any convex combination of these price sequences. But if the two price sequences were constant-rate-of-interest price sequences for different values of the rate of interest a convex combination of them will not be a constant-rate-ofinterest sequence and, as such, will be excluded from an analysis which confines its attention to constant-rate-of-interest sequences. [...]

Surely part of the excitement caused by the demonstration that double switching was seemingly a fairly 'common' phenomenon, in the sense that the examples do not depend upon choosing very special values for the parameters, arose not from the fact that a technique could be chosen by firms at more than one value of the rate of interest, an obvious consequence of the sparseness of a finite linear production model, but because it seemed that the set of values at which a technique might be chosen was not connected. But this is an optical illusion. Firms do not choose plans in the light of the rate of interest alone. They choose plans in the light of complete intertemporal price systems. [Bliss, 1975, p. 239, emphasis in the original]

The purpose of this note is to clarify the content of what Bliss maintained about reswitching without the complications of the intertemporal optimisation framework within which he set his arguments. ${ }^{1}$ This will be done by means of a very simple model with two commodities and three methods of production, which can be combined so as to have two techniques.

Section 2 considers the standard case in which the same stationary relative price for the two commodities is used on both the input and the output side. This case, whose properties are well-known, is then re-written in terms of ArrowDebreu intertemporal prices with some explicit conditions of stationarity imposed on them.

Section 3 shows that even though there is in general a convex set of intertemporal prices for which the two techniques can be used simultaneously, no set of prices satisfying the stationarity conditions is convex.

Finally, section 4 defines the sets of intertemporal prices supporting the use of each technique or the coexistence of both and their sub-sets satisfying the stationarity conditions. The main conclusion drawn from this analysis is that the

\footnotetext{
1 The point is also presented by Bliss in his entry on 'equal rates of profit' in the New Palgrave dictionary, but the argument there, being rapidly sketched, is less clear than in the passage quoted above (see Bliss 1990, p. 152).
} 
concept of a 'switch point' between two techniques makes no sense outside the hypothesis of stationary prices.

The question of whether the reswitching phenomenon entails negative repercussions for the modern general equilibrium theory, upon which various contributions already exist, ${ }^{2}$ is not discussed here.

\section{Stationary prices and reswitching}

Let us consider a model with two commodities, $x$ and $y$. Each of the commodities is both a consumption and a (circulating) capital good.

There are three methods of production, 1, 2 and 3:

$\begin{array}{ll}\text { [method 1] } & a_{11} \text { of } x \& a_{12} \text { of } y \& \ell_{1} \text { of labour } \rightarrow 1 \text { of } x \\ \text { [method 2] } & a_{21} \text { of } x \& a_{22} \text { of } y \& \ell_{2} \text { of labour } \rightarrow 1 \text { of } y \\ \text { [method 3] } & a_{31} \text { of } x \& a_{32} \text { of } y \& \ell_{3} \text { of labour } \rightarrow 1 \text { of } y\end{array}$

These three methods can be combined so as to obtain two techniques: technique $\alpha$, using methods 1 and 2 , and technique $\beta$, using 1 and $3 .^{3}$ Each technique is assumed to be viable and non-dominated.

On the assumption that the price of $y$ in terms of $x$, denoted by $\pi_{y}$, is stationary, meaning that it does not change period by period, the characteristics of the choice of technique for the case addressed here are well-known, and we shall therefore confine ourselves to recalling just some results. In particular, as an implication of the 'non-substitution theorem', it is known that for every given interest rate $r$, taken within a certain interval, the methods used are determined and the wage rate $w$ and the price $\pi_{y}$, corresponding to $r$, are determined accordingly [cf. Burmeister and Dobell (1970), ch. 8, theorem 8 (p. 242)].

\footnotetext{
2 On reswitching as a possible cause of equilibrium multiplicity or instability, see in particular Fratini (2007) and (2013), Garegnani (2003) and (2005), and Schefold (2005).

3 This model corresponds to the one considered by Garegnani in the appendix of his article for the 1966 symposium on the Quarterly Journal of Economics [see Garegnani (1966), pp. 566, 567].
} 
Moreover, up to two switch points are possible in the two-commodity, three-method framework considered here [cf. Burmeister and Dobell (1970), p. 249-50]. This means that the system:

$$
\begin{aligned}
& \left(a_{11}+a_{12} \cdot \pi_{y}\right)(1+r)+\ell_{1} \cdot w=1 \\
& \left(a_{21}+a_{22} \cdot \pi_{y}\right)(1+r)+\ell_{2} \cdot w=\pi_{y} \\
& \left(a_{31}+a_{32} \cdot \pi_{y}\right)(1+r)+\ell_{3} \cdot w=\pi_{y}
\end{aligned}
$$

can have two economically meaningful solutions. On the assumption that this is the case, if $\widehat{\pi}=\left[\widehat{r}, \widehat{w}, \widehat{\pi}_{y}\right]$ and $\breve{\pi}=\left[\breve{r}, \breve{w}, \breve{\pi}_{y}\right]$ are the price vectors solving the system, then one of the two techniques, say technique $\alpha$, is in use (optimal) for interest rates in the intervals $[0, \widehat{r}]$ and $[\breve{r}, R]$ - where $R$ is the maximum rate of interest compatible with a non-negative wage rate - but not in the interval $(\widehat{r}, \breve{r})$. In other words, the set of interest rates for which technique $\alpha$ is used is not convex.

All this can be graphically represented as in fig. 1, where there are the two wage-interest curves associated with techniques $\alpha$ and $\beta,{ }^{4}$ and, as is known, the technique used is the one that makes it possible to pay the maximum wage rate for a given rate of interest.

This is the usual way in which the reswitching argument is presented. It will, however, prove convenient for the purposes of the analysis developed in the following sections, to introduce some slight formal modifications that do not alter the substance of the matter.

First, instead of regarding the interest rate as the independent variable and the wage rate as the dependent, it is possible to do the opposite and in so doing to say that if $\widehat{\pi}=\left[\widehat{r}, \widehat{w}, \widehat{\pi}_{y}\right]$ and $\breve{\pi}=\left[\breve{r}, \breve{w}, \breve{\pi}_{y}\right]$ are the two solutions of the system (1)(3), then technique $\alpha$ is in use for wage rate levels in the intervals $[0, \widehat{w}]$ and $[\breve{w}, W]$ but not $(\widehat{w}, \breve{w})$, and therefore the set of wage rate levels for which technique $\alpha$ is in use is not convex.

\footnotetext{
${ }^{4}$ The wage-interest curve associated with technique $\alpha$ is the graph of the function $w_{\alpha}=w_{\alpha}(r)$, where $w_{\alpha}$ solves the equations (1) and (2) for a given $r$. In the same way, a function $w_{\beta}=w_{\beta}(r)$ can be defined by the equations (1) and (3).
} 
Fig. 1 - Wage-interest curves

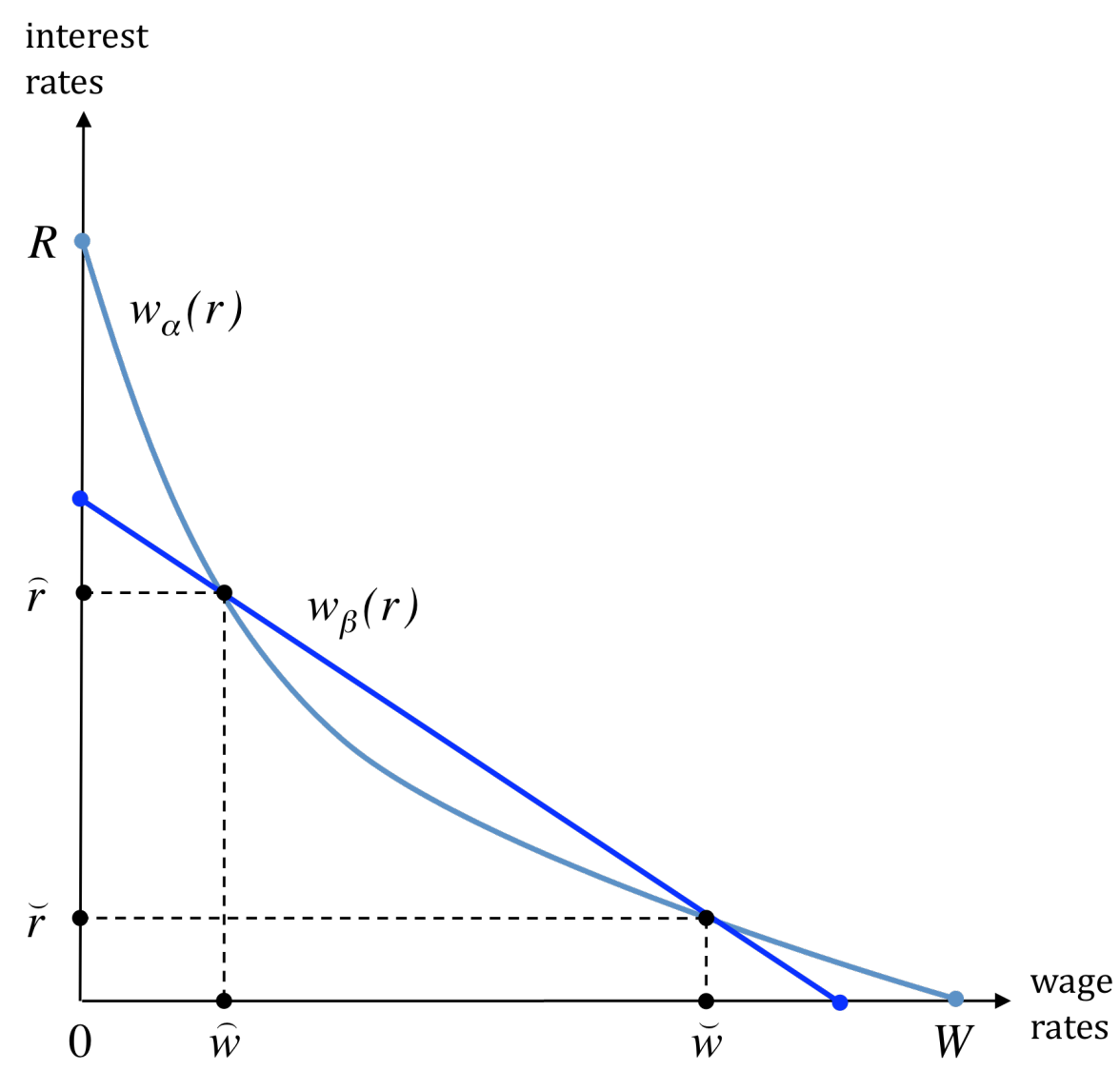

Second, on the basis of our hypothesis of stationary relative prices, $\pi_{y}$ is the price of $y$ in terms of $x$ on both the input and the output sides of equations (1)-(3), despite the fact that inputs are commodities delivered in period $t$ and outputs in period $t+1$. The hypothesis of stationary relative prices is implicit in equations (1)(3) but can be made explicit.

In particular, it is possible to follow the Arrow-Debreu methodology and distinguish commodities by their date of delivery and denote by $p_{i, j}$ the price of commodity $i$ delivered in period $j$, suitably actualised, with $i=x, y$ and $j=t, t+1$. Once this notation is introduced and the commodity $x$ delivered in $t+1$ is assumed as the numéraire, i.e. $p_{x, t+1}=1$ is posited, the following equality holds in the case of stationary relative prices considered here: 


$$
\frac{p_{y, t}}{p_{x, t}}=p_{y, t+1}=\pi_{y}
$$

which can also be written as follows: ${ }^{5}$

$$
p_{x, t}=\frac{p_{y, t}}{p_{y, t+1}}=1+r
$$

It is in fact easy to prove that equalities (4) and (5) together with the system

$$
\begin{aligned}
& a_{11} \cdot p_{x, t}+a_{12} \cdot p_{y, t}+\ell_{1} \cdot w=1 \\
& a_{21} \cdot p_{x, t}+a_{22} \cdot p_{y, t}+\ell_{2} \cdot w=p_{y, t+1} \\
& a_{31} \cdot p_{x, t}+a_{32} \cdot p_{y, t}+\ell_{3} \cdot w=p_{y, t+1}
\end{aligned}
$$

make it possible to obtain the usual system (1)-(3).

Finally, since the systems (1)-(3) and (4)-(8) are actually equivalent, if the former has two solutions, as is assumed here, then so does the latter. ${ }^{6}$

\section{Stationary prices and non-convexity}

Given the two solutions of system (1)-(3), namely $\widehat{\pi}=\left[\widehat{r}, \widehat{w}, \widehat{\pi}_{y}\right]$ and $\breve{\pi}=\left[\breve{r}, \breve{w}, \breve{\pi}_{y}\right]$, there are two intertemporal price vectors associated with them, namely

\footnotetext{
${ }^{5}$ By definition, the own-rates of interest of the two commodities are: $r_{x}=p_{x, t} / p_{x, t+1}-1$ and $r_{y}=$ $p_{y, t} / p_{y, t+1}-1$. It is therefore immediately evident that $r_{x}=r_{y}=r$ if and only if $p_{y, t} / p_{x, t}=p_{y, t+1} / p_{x, t+1}=\pi_{y}$. Therefore, in a stationary price vector, "all present-value price vectors [are] proportional to each other and all own-rates of interest equal" (Bliss, 1975, p. 69). On this point, see also Bliss (1990), p. 151.

${ }^{6}$ Given a solution $\hat{\pi}=\left[\hat{r}, \widehat{w}, \hat{\pi}_{y}\right]$ of system (1)-(3), equalities (4) and (5) make it possible to determine the corresponding prices $\hat{p}_{x, t}, \hat{p}_{y, t}$ and $\hat{p}_{y, t+1}$ univocally. In particular, we have: $\hat{p}_{x, t}=1+\widehat{r} ; \hat{p}_{y, t}=\widehat{\pi}_{y}(1+\widehat{r})$ and $\hat{p}_{y, t+1}=\widehat{\pi}_{y}$. Therefore, since $\hat{\pi}=\left[\widehat{r}, \widehat{w}, \hat{\pi}_{y}\right]$ solves the system (1)(3), then $\hat{p}=\left[\hat{p}_{x, t}, \hat{p}_{y, t}, \widehat{w}, 1, \hat{p}_{y, t+1}\right]$ satisfies equations (6)-(8).
} 
$\hat{p}=\left[\widehat{p}_{x, t}, \widehat{p}_{y, t}, \widehat{w}, 1, \hat{p}_{y, t+1}\right]$ and $\breve{p}=\left[\breve{p}_{x, t}, \breve{p}_{y, t}, \breve{w}, 1, \breve{p}_{y, t+1}\right]$, that solve system (6)-(8) and satisfy conditions (4) and (5). ${ }^{7}$

Now, since system (6)-(8) is linear, it is clear that every convex linear combination of $\hat{p}$ and $\breve{p}$ is again one of its possible solutions. In other words, every $\hat{p}=\theta \hat{p}+(1-\theta) \breve{p}$, with $0<\theta<1$, solves the system. With $\hat{p} \neq \breve{p}$, however, $\hat{p}$ does not satisfy conditions (4) and (5). This can be easily proved.

Proposition 1. Let $p^{\prime}$ and $p^{\prime \prime}$ be two stationary intertemporal price vectors associated with $\pi_{y}{ }^{\prime} \neq \pi_{y}{ }^{\prime \prime}$ and $r^{\prime} \neq r^{\prime \prime}$. Any price vector $p^{\prime \prime \prime}=\theta p^{\prime}+(1-\theta) p^{\prime \prime}$, with $0<\theta$ $<1$, is not a stationary price vector.

Proof. Since $p^{\prime}$ and $p^{\prime \prime}$ are stationary intertemporal price vectors, according to equation (4) we have: $p_{y, t}^{\prime} / p_{x, t}^{\prime}=p_{y, t+1}^{\prime}$ and $p_{y, t}^{\prime \prime} / p_{x, t}^{\prime \prime}=p_{y, t+1}^{\prime \prime}$. This implies: $p^{\prime \prime \prime}{ }_{y, t+1}=\theta$ $p_{y, t}^{\prime} / p_{x, t}^{\prime}+(1-\theta) p_{y, t}^{\prime \prime} / p_{x, t}^{\prime \prime}$. Therefore, since $p_{y, t}^{\prime \prime \prime} / p_{x, t}^{\prime \prime}=\left[\theta p_{y, t}^{\prime}+(1-\theta) p_{y, t}^{\prime \prime}\right] /\left[\theta p_{x, t}^{\prime}+\right.$ $\left.(1-\theta) p_{x, t}^{\prime \prime}\right]$, the price vector $p^{\prime \prime \prime}$ can be stationary if and only if:

$$
\theta \frac{p_{y, t}^{\prime}}{p_{x, t}^{\prime}}+(1-\theta) \frac{p_{y, t}^{\prime \prime}}{p_{x, t}^{\prime \prime}}=\frac{\theta p_{y, t}^{\prime}+(1-\theta) p_{y, t}^{\prime \prime}}{\theta p_{x, t}^{\prime}+(1-\theta) p_{x, t}^{\prime \prime}}
$$

After some simple algebraic steps, this can also be written as

$$
\left(\pi_{y}{ }^{\prime}-\pi_{y}{ }^{\prime \prime}\right)\left(r^{\prime \prime}-r^{\prime}\right)=0
$$

which is, however, impossible as $\pi_{y}^{\prime} \neq \pi_{y}{ }^{\prime \prime}$ and $r^{\prime} \neq r^{\prime \prime}$.

This result has some interesting implications. First, with attention focused solely on the stationary prices, the levels of the wage rate at which the two techniques are simultaneously in use are isolated points: the switch points. If instead non-stationary intertemporal price vectors are also admitted, it is not possible to talk about switch points because the two techniques can be

\footnotetext{
7 This clearly means that $\hat{p}_{y, t} / \widehat{p}_{x, t}=\hat{p}_{y, t+1}=\hat{\pi}_{y}$ and $\hat{p}_{x, t}=\hat{p}_{y, t} / \hat{p}_{y, t+1}=1+\widehat{r}$; and similarly $\breve{p}_{y, t} / \breve{p}_{x, t}=\breve{p}_{y, t+1}=\breve{\pi}_{y}$ and $\breve{p}_{x, t}=\breve{p}_{y, t} / \breve{p}_{y, t+1}=1+\breve{r}$. See also the previous footnote.
} 
simultaneously in use for every wage rate level between 0 and a certain maximum, provided that the other prices are adjusted according to equations (6)-(8). ${ }^{8}$

Second, as shown by Proposition 1, in the case of two intertemporal price vectors that are stationary, any price vector obtained as a convex linear combination of them is not stationary. Consequently, any set of stationary intertemporal prices with more than one element cannot be a convex set.

Third, combining the previous two remarks, it is useful to distinguish two different kinds of non-convexity that can arise when the analysis is restricted to price vectors that are stationary, i.e. that satisfy conditions (4) and (5). There is the non-convexity of the wage rate levels at which a certain technique - technique $\alpha$ in the case considered here - is used due to the possibility of reswitching. There is, however, also the non-convexity of the set of stationary price vectors at which each technique - both $\alpha$ and $\beta$ - is used due to the fact that no set of stationary price vectors can be convex, and this is independent of the reswitching phenomenon.

\section{Sets of price vectors}

Attention has been focused so far primarily on price vectors that allow the two techniques to be used simultaneously. It is now possible to broaden our analysis to obtain a more general view.

\footnotetext{
${ }^{8}$ The proof of the possibility of non-negative prices satisfying the system (6)-(8) with a null wage rate can be briefly outlined. With $w=0$, equation (6) implies $p_{y, t}=1 / a_{12}-\left(a_{11} / a_{12}\right) p_{x, t}$, which is the equation of a decreasing straight line crossing the vertical axis at point $1 / a_{12}$. Equations (7) and (8) instead bring about $p_{y, t}=-\left(a_{21}-a_{31}\right) /\left(a_{22}-a_{32}\right) p_{x, t}$ which is the equation of a straight line starting from the origin of the axes. If therefore, in the production of $y$, one of the two alternative methods employs more of commodity $x$ per unit of output and the other more of commodity $y$, then the straight line derived from equations (7) and (8) is increasing and consequently intersects the other line for a pair of strictly positive prices $\left(p_{x, t}, p_{y, t}\right)$. And this also implies $p_{y, t+1}>0$.

If instead the method of production of $y$ that employs more of commodity $x$ per unit of output employs more of commodity $y$ too, then there is a minimum strictly positive level of $w$ compatible with the coexistence of techniques. There is a very simple reason for this, namely that the method employing both less of $x$ and less of $y$ per unit of output is unquestionably the most advantageous when $w=0$. This appears, however, to be a particular case in a certain sense, as it allows us to say which technique is more capital-intensive than the other through direct reference to physical capital without knowing the prices.
} 
Since no activity can entail strictly positive profits in equilibrium, let us start from the set $P$, whose elements are intertemporal price vectors satisfying the following conditions:

$$
\begin{aligned}
& a_{11} \cdot p_{x, t}+a_{12} \cdot p_{y, t}+\ell_{1} \cdot w \geq 1 \\
& a_{21} \cdot p_{x, t}+a_{22} \cdot p_{y, t}+\ell_{2} \cdot w \geq p_{y, t+1} \\
& a_{31} \cdot p_{x, t}+a_{32} \cdot p_{y, t}+\ell_{3} \cdot w \geq p_{y, t+1} .
\end{aligned}
$$

If $P_{\alpha}$ is the set of price vectors for which the use of technique $\alpha$ is optimal, then $P_{\alpha} \subset P$ is made up of price vectors satisfying conditions (11) and (12) with equality. In fact, since, for every $p \in P$, no activity can bring about strictly positive profits, then the optimal technique entails zero profits. In the same way, the set $P_{\beta}$ $\subset P$ contains price vectors for which conditions (11) and (13) are satisfied with equality. ${ }^{9}$ Elements of the set $P_{\alpha \beta}=P_{\alpha} \cap P_{\beta}$ are clearly the price vectors for which the two techniques can be used simultaneously, i.e. price vectors solving the system (6)-(8).

There are price vectors that are stationary in each of these sets. In particular, let $\tilde{P}_{\alpha}, \tilde{P}_{\beta}$ and $\tilde{P}_{\alpha \beta}$ be the sub-sets of $P_{\alpha}, P_{\beta}$ and $P_{\alpha \beta}$ whose elements satisfy the stationarity conditions (4) and (5).

Since conditions (11)-(13) are linear, the sets $P_{\alpha}, P_{\beta}$ and $P_{\alpha \beta}$ are convex. On the contrary, Proposition 1 implies that the sets $\tilde{P}_{\alpha}$ and $\tilde{P}_{\beta}$ are always nonconvex. As regards the set $\tilde{P}_{\alpha \beta}$, its elements are the switch points and therefore, in the case addressed here, it can have (generically) ${ }^{10}$ one or two elements. In the latter case, it is non-convex.

With regard to the set of stationary prices $\tilde{P}_{\alpha} \cup \tilde{P}_{\beta}$, there is a one-to-one correspondence between its elements and wage rates in the interval $[0, W]$.

\footnotetext{
${ }^{9}$ It is clear that $P_{\alpha} \cup P_{\beta} \subset P$, and also that the set $P \backslash\left\{P_{\alpha} \cup P_{\beta}\right\}$ is non-empty.

10 The case in which the wage-interest curves of the two techniques coincide on a certain stretch is ignored here because it is associated with a non-generic choice of technical coefficients.
} 
Proposition 2. For any given $w^{\prime} \in[0, W]$, there is one and only one price vector $p^{\prime}=\left[p_{x, t}^{\prime}, p_{y, t}^{\prime}, w^{\prime}, 1, p_{y, t+1}^{\prime}\right] \in P_{\alpha} \cup P_{\beta}$ that satisfies the stationarity conditions (4) and (5).

Proof. As noted above in section 2, for every given rate of interest $r \in[0, R]$, the methods of production used and the corresponding (zero-profit) price system $\pi=\left[r, w, \pi_{y}\right]$ are determined. Moreover, since there is a one-to-one correspondence between $w$ and $r$ under stationary conditions (the "wage-interest frontier") then, given $w^{\prime} \in[0, W]$, there is one and only one stationary price system $\pi^{\prime}=\left[r^{\prime}, w^{\prime}, \pi_{y}^{\prime}\right]$ associated with $w^{\prime}$.

Finally, given $\pi^{\prime}=\left[r^{\prime}, w^{\prime}, \pi_{y}^{\prime}\right]$, conditions (4) and (5) make it possible to determine univocally the prices $p_{x, t}^{\prime}, p_{y, t}^{\prime}$ and $p_{y, t+1}^{\prime}$ associated with $r^{\prime}$ and $\pi_{y}^{\prime}$.

To sum up, if non-stationary prices are ruled out, there is a unique price vector associated with any given level of the wage rate. In this context, talking about "switch points" makes perfect sense, because the methods in use are determined once the wage rate level is known, and wage rate levels for which the two techniques can coexist are (generically) isolated points.

If instead non-stationary intertemporal price vectors are admitted, then there is a point-set correspondence between wage rate levels and vectors in $P_{\alpha} \cup P_{\beta}$. In particular, given $w^{\prime} \in[0, W], 11$ it is very possible for a set of intertemporal price vectors paying a wage rate $w^{\prime}$ to include: a) some price vectors entailing the use of technique $\alpha$ alone; b) other price vectors at which technique $\beta$ only is in use; and c) a price vector allowing both the techniques to be simultaneously used. ${ }^{12}$ As a result, if the analysis is not restricted to stationary price sets, there are no "switch points" because the two techniques can be used

\footnotetext{
${ }^{11}$ It can be observed in passing that in the case of stationary prices, wage rate levels above $W$ are regarded as economically inadmissible because they bring about negative interest rates $r$. When non-stationary price vectors are instead admitted, it seems possible to have vectors of non-negative prices associated with wage rates higher than $W$. This possibility appears, however, to play no role in the argument presented in this paper.

${ }^{12}$ To see this it is enough to observe that, once $w$ is taken as given, there are three unknown prices in the system (11)-(13) and therefore its degrees of freedom depend on the number of conditions satisfied with the inequality sign.
} 
simultaneously for every wage rate level in a certain interval, provided that the other prices are properly adjusted.

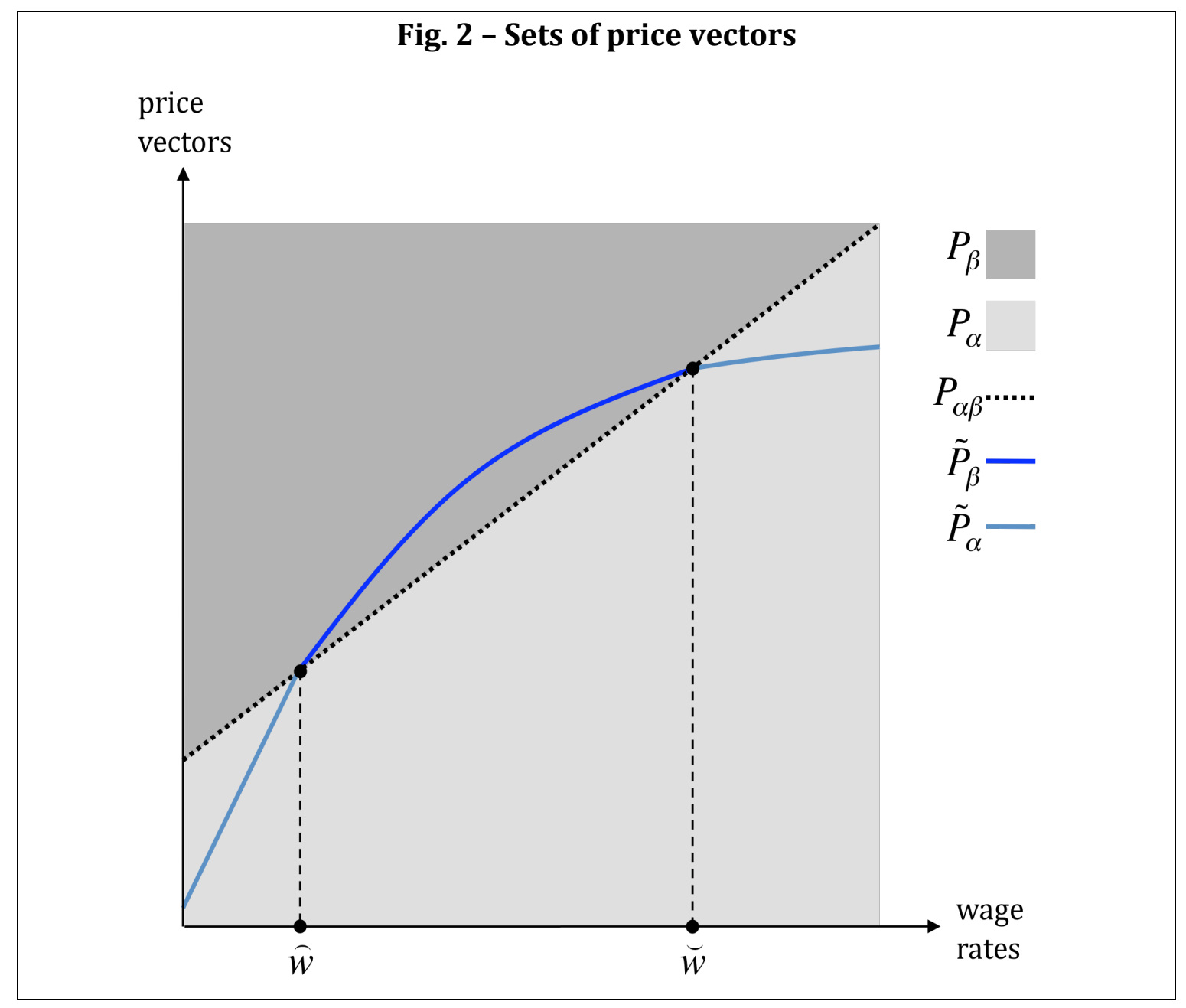

All these considerations about price sets and wage rate levels can be exemplified - albeit not properly represented - by means of a graphic diagram with wage rate levels on one axis and price vectors on the other, ${ }^{13}$ as in fig. 2 .

In fig. 2 , the set of stationary intertemporal price vector $\tilde{P}_{\alpha} \cup \tilde{P}_{\beta}$ is represented by a line (light and dark blue), since, as seen above, there is a one-toone correspondence between its elements and wage rate levels. Moreover, it is clear that the set $\tilde{P}_{\alpha} \cup \tilde{P}_{\beta}$ is not convex and therefore that no linear convex

13 The idea for this kind of graphic representation is taken from fig. 17.D.6 in Mas-Colell, Winston and Green (1995, p. 598), where points on the vertical axis are assumed to be vectors of $L-1$ prices. 
combination of two stationary intertemporal price vectors is a vector of stationary prices.

The sets $P_{\alpha}$ (light gray), $P_{\beta}$ (dark gray) and $P_{\alpha \beta}$ (dotted line) are instead convex.

The two intersections between the sets $\tilde{P}_{\alpha} \cup \tilde{P}_{\beta}$ and $P_{\alpha \beta}$ are the switch points.

\section{Conclusions}

On the basis of the analysis put forward in this paper, there is no doubt that the possibility of reswitching is restricted to cases in which the stationarity of relative prices is assumed.

This conclusion follows primarily from the fact that, as we have seen, the concept of a switch point between two techniques loses its meaning outside the case of stationary prices. On the one hand, if non-stationary price vectors are admitted, then two techniques can be simultaneously used for every wage rate taken in a certain interval. On the other, it is under stationarity conditions alone that a wage rate level (taken in a certain interval) can be univocally associated with a price vector (Prop. 2) so that the technique used is simultaneously known. In the non-stationary case, on the contrary, many different price vectors, supporting the use of different techniques, can be associated with the same wage rate.

Moreover, no set of stationary intertemporal price vectors is convex (Prop. 1). The role of this fact in the reswitching phenomenon is not clear. It implies that the set of price vectors associated with the switch points between two techniques is made up of isolated points, but the central issue is whether this set has more than one element, and non-convexity does not appear to have much to do with this.

Finally, since reswitching requires the stationarity of relative prices period by period, it is obvious that it cannot take place in a framework where stationarity is inconceivable, such as atemporal equilibrium. 


\section{Acknoledgements}

Sicere thanks are due to Christian Gehrke and an anonimous referee for their comments and suggestions. The usual disclaimer applies for any remaining errors.

\section{References}

Bliss, C.J. (1975) Capital Theory and Distribution of Income, Amsterdam/New York: North-Holland/Elsevier.

Bliss, C.J. (1990) 'Equal rates of profit', in J. Eatwell, M. Milgate, P. Newman (eds) Capital Theory, London: Macmillan.

Burmeister, E. and Dobell, A.R. (1970) Mathematical Theories of Economic Growth, London: Macmillan.

Fratini, S.M. (2007) 'Reswitching of techniques in an intertemporal equilibrium model with overlapping generations', Contributions to Political Economy, 26(1), pp. 43-59.

Fratini, S.M. (2013) 'Real Wicksell effect, demand for capital and stability', Metroeconomica, 64(2), pp. 346-360.

Garegnani, P. (1966) 'Switching of techniques', The Quarterly Journal of Economics, 80(4), pp. 554-567.

Garegnani, P. (2003) 'Saving, investment and the quantity of capital in general intertemporal equilibrium', in F. Hahn and F. Petri (eds) General Equilibrium. Problems and Prospects, London: Routledge.

Garegnani, P. (2005) 'Capital and intertemporal equilibria: A reply to Mandler', Metroeconomica, 56(4), pp. 411-437.

Mas-Colell, A.; Whinston, M.D. \& Green, J. (1995) Microeconomic Theory, Oxford: Oxford University Press.

Schefold, B. (2005) 'Reswitching as a cause of instability of intertemporal equilibrium', Metroeconomica, 56(4), pp. 438-476. 